\title{
Revalidación taxonómica y distribución potencial de Armatocereus brevispinus Madsen (Cactaceae)
}

Taxonomic revalidation and potential distribution of Armatocereus brevispinus Madsen (Cactaceae)

\section{Christian R. Loaiza S. ${ }^{1 *}$ y José Roque Gamarra ${ }^{2}$}

1 Departamento de Mastozoología, Museo de Historia Natural, Universidad Nacional Mayor de San Marcos, Av. Arenales 1256, Jesús María, Lima, Perú. 2 Laboratorio de Florística, Museo de Historia Natural, Universidad Nacional Mayor de San Marcos, Av. Arenales 1256, Jesús María, Lima, Perú.

*Autor para correspondencia

Email Christian Loaiza: christian.loaiza@unmsm.edu.pe

Email José Roque: jroqueg@unmsm.edu.pe

ORCID José Roque: http://orcid.org/0000-0001-6840-6603

\begin{abstract}
Resumen
Se reporta el primer registro confirmado para el norte de Perú de Armatocereus brevispinus Madsen, una especie de cactácea considerada como endémica de la provincia de Loja, en la región sur del Ecuador. El registro corresponde a una localidad situada en el km 18 de la vía a Ayabaca (04 42' 21.0" S y 79 48' 51.0" W), en el distrito de Ayabaca, dentro de la provincia de Ayabaca, en el Departamento de Piura. Este registro representa una ampliación del rango distributivo conocido para esta especie en Ecuador. Se analiza la taxonomía, distribución y el estado de conservación y se comenta sobre su biogeografía en la región andina.
\end{abstract}

Palabras clave: Armatocereus; distribución; endemismo; Piura; Perú; Ecuador.

\section{Abstract}

We report the first confirmed record for northern Peru of Armatocereus brevispinus Madsen, a species of cactus regarded as endemic to the province of Loja, in the southern region of Ecuador. The record corresponds to a locality placed near the $\mathrm{km} 18$ of the route to Ayabaca $\left(04^{\circ} 42^{\prime} 21.0^{\prime \prime S}\right.$ and $\left.79^{\circ} 48^{\prime} 51.0^{\prime \prime} \mathrm{W}\right)$, in the district of Ayabaca, within the province of Ayabaca, in the Department of Piura. This records represents an extension of the distributional range known for this species in Ecuador. We analyze its taxonomy and the distribution and conservation status and comments on its biogeography in the Andean region.

Keywords: Armatocereus; distribution; endemism; Piura; Perú; Ecuador.

\section{Citación:}

Loaiza C.R. \& J. Roque Gamarra. 2016. Revalidación taxonómica y distribución potencial de Armatocereus brevispinus Madsen (Cactaceae). Revista peruana de biología 23(1): 035 - 041 (Abril 2016). doi: http:// dx.doi.org/10.15381/rpb.v23i1.11831

Fuentes de financiamiento:

Los autores declaran no tener financiamiento

Presentado: $\quad$ 20/12/2015

Aceptado: $\quad 15 / 03 / 2016$

Publicado online: $28 / 05 / 2016$
Información sobre los autores:

CLS realizó la revisión de las colecciones del Herbario San Marcos, confirmó la validez de la especie y preparó el primero borrador del manuscrito; JRG corroboró la determinación taxonómica de la especie, realizó la fotografía del voucher y realizó una revisión crítica del manuscrito. Los autores no incurren en conflictos de intereses. 


\section{Introducción}

La familia Cactaceae es endémica de América (Anderson 2001). Su distribución comprende todo el continente, desde el nivel del mar hasta las zonas altoandinas (Nyffeler 2002, Arakaki 2011). En Perú, esta familia se encuentra representada por 40 géneros y 262 especies (Ostolaza 2014).

El género Armatocereus es el único género de cactus endémico de la ecorregión Tumbesina, presente tanto en Ecuador como en Perú (Madsen 1989, Ostolaza 2006, 2014). Son cactáceas que poseen junto a otros géneros (Jasminocereus y Weberocereus) características primitivas de la familia, como la presencia de espinas en flores y frutos (Backeberg 1938, Ritter 1981). Su nombre se deriva del latín armatus (armado) y cereus (cirio), que hace referencia a su forma columnar y en alusión a sus fuertes espinas (Madsen 1989, Ostolaza 2006).

Este género se consideraba representado por alrededor de 10 especies nominales distribuidas en Perú, Ecuador y Colombia (Madsen 1989). La única especie atribuida a Colombia (Armatocereus humilis) presente en Dagua (valle del Cauca), actualmente está incluida dentro del género Stenocereus (Hunt 2002). De acuerdo a los últimos estudios y revisiones realizadas en Perú y Ecuador, este género estaría representado por once especies y dos subespecies, distribuidas en el centro y suroccidente de Ecuador y en el noroccidente del Perú (Loaiza 2013, Ostolaza 2006). Según la última clasificación propuesta por Ostolaza (2014), cuatro especies se encuentran presentes en Ecuador, de las cuales tres están compartidas con el norte de Perú: $A$. cartwrightianus, $A$. laetus y $A$. rupicola. Loaiza (2013) valida y reconoce la especie descrita como A. brevispinus por Madsen (1989), la cual no es reconocida por Ostolaza (2014). Las nueve especies adicionales están catalogadas como endémicas de algunos biomas del Perú como el Bosque Seco Ecuatorial y el Desierto del Pacífico (BrackEgg 1986, Ostolaza 2006, 2014).

Este género está representado por plantas columnares, arbóreas o de tipo arbustivas, de hasta $12 \mathrm{~m}$ de altura, con ramas cilíndricas, ascendentes y articuladas debido al crecimiento anual (Backeberg 1938). Tallos formados por segmentos de 30 - $70 \mathrm{~cm}$ de largo, y provistos de 4 - 12 costillas muy marcadas (Madsen 2002, Ostolaza 2006). Las areolas de las partes florales muy similares a las de las partes no florales (Madsen 1989). Espinas gruesas y fuertes, rara vez escasas o ausentes (Ostolaza 2006). Flores nocturnas y tubulares (7 - $12 \mathrm{~cm}$ de largo), con

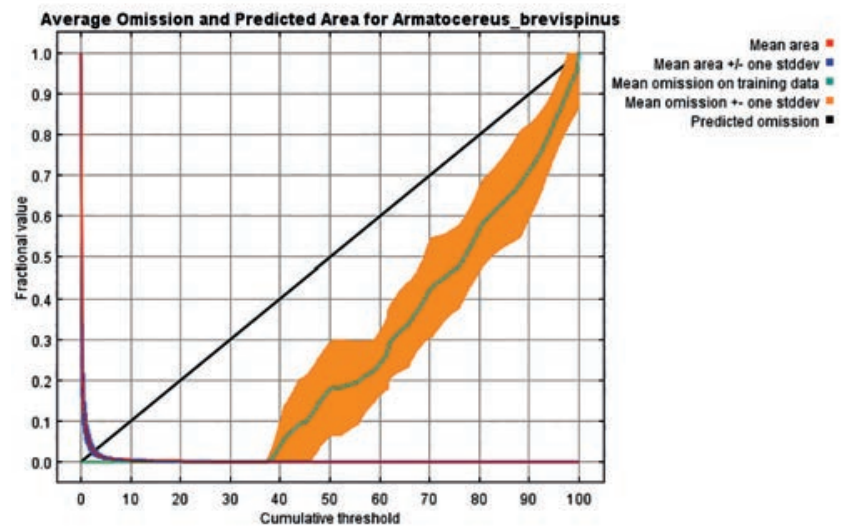

Figura 1. Modelo de curva acumulativa (AUC) para Armatocereus brevispinus el tubo floral y ovario espinosos; el perianto es de color blanco (rojo solo en $A$. rauhii) (Ostolaza 2006). Algunas especies son consideradas como plantas polinizadas por murciélagos (Simmons y Wetterer 2002). Los frutos son grandes $(5-13 \mathrm{~cm}$ de largo), de forma globosa a ovoide, rojos o verdes, fuertemente espinosos, con espinas que suelen perder al madurar (Madsen 1989). Las semillas son grandes, negras, ovoides o reniformes, según la especie (Ostolaza 2006).

La probabilidad de la presencia de $A$. brevispinus en los bosques secos del norte de Perú ya había sido señalado anteriormente por Loaiza (2013), por lo cual quedaba la inquietud de poder comprobar esta hipótesis a futuro en base a revisión de material botánico depositado en los principales herbarios de Perú o también por registro directo en las zonas limítrofes con Ecuador, en el norte de Perú.

\section{Materiales y métodos}

Modelamiento distributivo.- Para analizar la distribución potencial de esta especie se utilizó el programa Maxent, el cual permite realizar modelos de máxima entropía, es decir, distribuciones de probabilidad en una forma sistemática y objetiva (Phillips et al. 2006). Este programa estima la probabilidad de ocurrencia de una especie buscando la distribución de máxima entropía (lo más uniforme posible), sujeta a la condición de que el valor esperado de cada variable ambiental, según esta distribución, coincida con su media empírica (Merow et al. 2013, Morales 2012, Phillips \& Dudík 2008, Riquelme 2013).

Para obtener la distribución potencial de esta especie se construyó una base de datos en Excel, la cual contó con nueve registros. Esta información posteriormente fue georeferenciada y transformada a un archivo de texto CSV (delimitado por comas). Debido al escaso números de registros disponibles, se desarrollaron 10 réplicas para el modelo, con el 25\% de los registros usados como test data, 500 iteraciones y 10000 puntos de respaldo "background points" con la finalidad de obtener una mayor precisión en el modelamiento. Esta metodología se aplicó para hacer un "jackniffe" y determinar las variables ambientales que mejor contribuyen con el modelamiento (Figs. 1 y 2). Luego de registrar las variables ambientales con menor valor de contribución con el modelamiento, se corrió nuevamente el modelamiento y se seleccionó como archivo de salida "output" un modelo logístico, ya que este modelo es el más fácil de conceptualizar y permite obtener como resultado

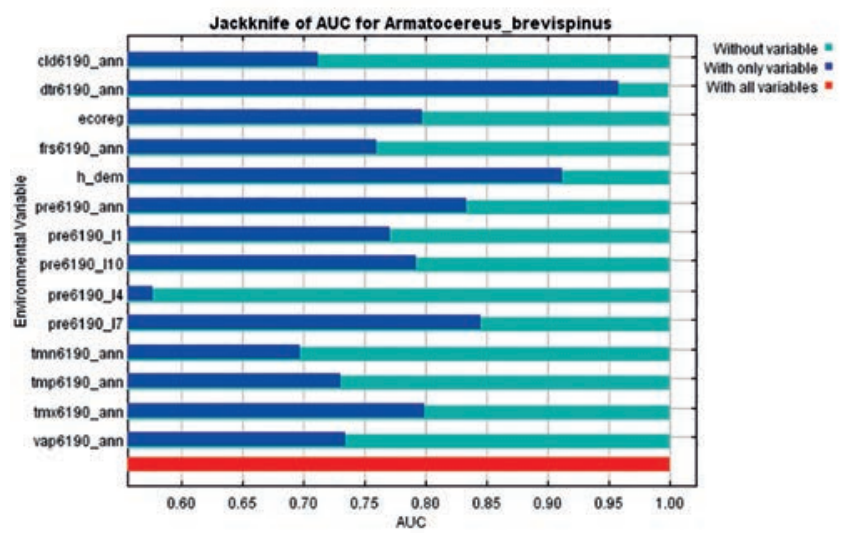

Figura 2. Test de jackknife realizado para determinar la importancia de las variables en el modelamiento 
Tabla 1. Resultados de las áreas predictivas para los valores de corte acumulativo 1, 5, 10 y valor de corte mínimo generados para el modelamiento de Armatocereus brevispinus.

\begin{tabular}{ccc}
\hline \multirow{2}{*}{$\begin{array}{c}\text { Valores de corte } \\
\text { acumulativo }\end{array}$} & \multicolumn{2}{c}{ Modelamiento } \\
\cline { 2 - 3 } & $\begin{array}{c}\text { Fraccional de área } \\
\text { predictiva }\end{array}$ & $\begin{array}{c}\text { Tasa de omisión } \\
\text { de formación }\end{array}$ \\
\hline 1 & 0.145 & 0 \\
5 & 0.014 & 0 \\
10 & 0.007 & 0 \\
Mínimo & 0.002 & 0 \\
\hline
\end{tabular}

un mapa binario, con valores entre 0 y 1 , en donde 1 es el valor que permite visualizar el área de distribución de una especie.

El propósito de la gráfica de la curva operacional (Curva ROC) obtenida para el modelamiento es señalar, en el caso de la curva roja (entrenamiento), el ajuste del modelo a los datos del muestreo y, en el caso de la curva azul (test), el grado de ajuste del modelo a los datos y supone el test real del poder predictivo del modelo. La línea negra representa a su vez, la línea esperada si el modelo no fuese mejor que "por azar". El valor del AUC representado en la curva $\mathrm{ROC}$ va de 0 a 1 y se considera que mientras más cerca se encuentre el valor obtenido a 1 , mejor es el criterio de aceptación del modelamiento obtenido.

Como análisis estadístico, se evaluaron los test dependiente e independiente, usando para ello como umbral de corte los valores de P en 1, 5 y 10 bajo el área de curva AUC (Tabla 1). Para el umbral de corte, se seleccionó la opción para el mínimo de presencia de los puntos de entrenamiento "Minimum training presence", lo cual permite obtener modelos bien precisos y además, permite generar como resultado archivos en formato "shapefile" que permiten realizar mapas distributivos.

Material examinado.- 1. Kilómetro 12 de la vía Catamayo - Loja, 79¹9'W y 03059'S (1950 - 2000 m), Madsen et al.: 75217, 75633, 75910, 86070 y 89128 (AAU, K, LOJA, MO, QCA, QCNE). 2. Vía Celica - El Empalme, 7958'W y 0407'S (1850 m), Jens E. Madsen y Orlando A. Sánchez: 7172 y 7173 (AAU, LOJA, QCNE y ZSS). 3. Km. 6 de la vía Catacocha Loja, 79³8'W y 04º4'S (1800 m), Jens E. Madsen y Orlando A. Sánchez: 7547 (AAU, LOJA, MO, QCA, QCNE, ZSS). 4. Kilómetro 10 de la vía Catacocha - Catamayo, 79³5’W y 040'ㅇ (1900 - 2000 m), Madsen et al.: 8248 (AAU, LOJA, MO, QCA, QCNE, ZSS). 5. Laderas del cerro Ahuaca (Cariamanga), 79³2'W y 04\%18'S (1934 m), Christian R. Loaiza S.: CL - 021, CL - 022 y CL - 023 (HUTPL). 6. Sector de Langunche (Paltas), 7948'W y 04 04'S (932 m), Christian R. Loaiza S.: CL - 026 (HUTPL).

Nuevo registro.- La muestra de herbario analizada corresponde a un registro realizado por Paul C. Hutchison y J. Kenneth Wright en 1964 (Fig. 3), el cual se encuentra depositado en el herbario San Marcos (USM) del Museo de Historia Natural de la Universidad Nacional Mayor de San Marcos, con el número de código de colección 6670. La localidad del registro corresponde a una zona de matorral seco montano situada en el km 18 de la vía a Ayabaca, en el distrito de Ayabaca, dentro de la provincia de Ayabaca, en el Departamento de Piura. Las coordenadas del registro son: 79048'51.0"W y 04042'21.0"S, y la altitud es sobre los $1700 \mathrm{~m}$. El espécimen fue identificado durante una revisión

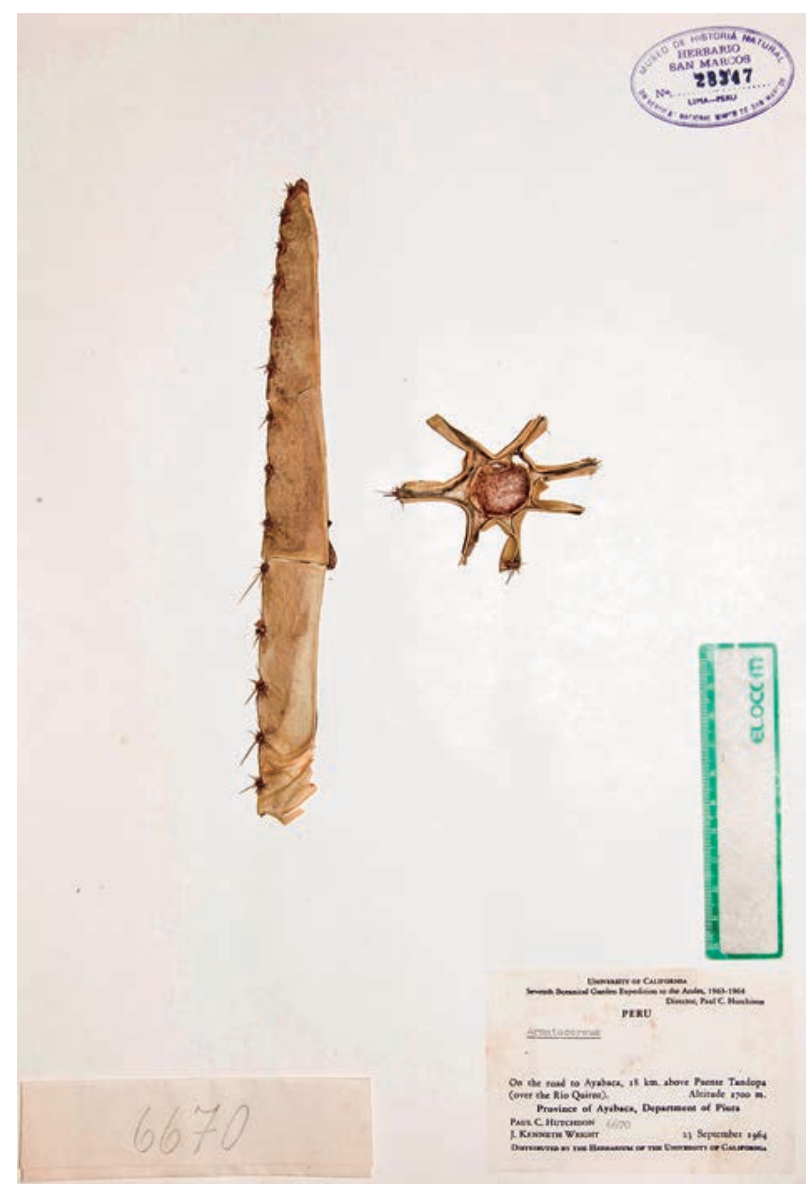

Figura 3. Ejemplar de $A$. brevispinus, Hutchison \& Wright 6670, colectado en 1964 y depositado en el Herbario San Marcos (USM).

de las colecciones del género Armatocereus realizada por el primer autor de este trabajo. La muestra solo había sido identificada a nivel de género y estaba en buen estado de conservación, lo cual permitió su correcta identificación en base a la forma y disposición de las espinas.

\section{Resultados}

\section{Armatocereus brevispinus Madsen}

Descripción.- Planta arbórea, de 8 a $10 \mathrm{~m}$ de alto, con el tronco leñoso y maderable, entre $15-50 \mathrm{~cm}$ de ancho, medianamente ramificado y poco espinoso (Fig. 4). Los segmentos del tallo son de color verde oscuro con diversas estrecheces notablemente marcadas, debido al crecimiento anual, de 40 - 80 $\mathrm{cm}$ de largo x $7-12 \mathrm{~cm}$ de ancho. El número de costillas varía entre 4 y 8 . Las areolas son de forma ampliamente ovalada, de 4 - $7 \mathrm{~mm}$ de ancho y de color café lanoso claro, separadas entre sí por $2-5 \mathrm{~cm}$. La espina central es de tamaño reducido, de 2 - $7 \mathrm{~mm}$ de largo con la punta de color oscuro. Espinas radiales (7 - 10) por areola, desplazándose en distintas direcciones. Flores solitarias, en forma de embudo, dispuestas de forma horizontal, de $8 \mathrm{~cm}$ de largo $\times 6 \mathrm{~cm}$ de ancho, con los sépalos de color verde, glabros y recurvados, y los pétalos de color blanco, con el ápice redondeado. Las brácteas estrechamente deltoides, inconspicuas, de 1 - 5 mm de largo. El fruto presenta forma ovoide, de 7 - 10 $\mathrm{cm}$ de largo, de color verde, recubierto por espinas de tamańo 


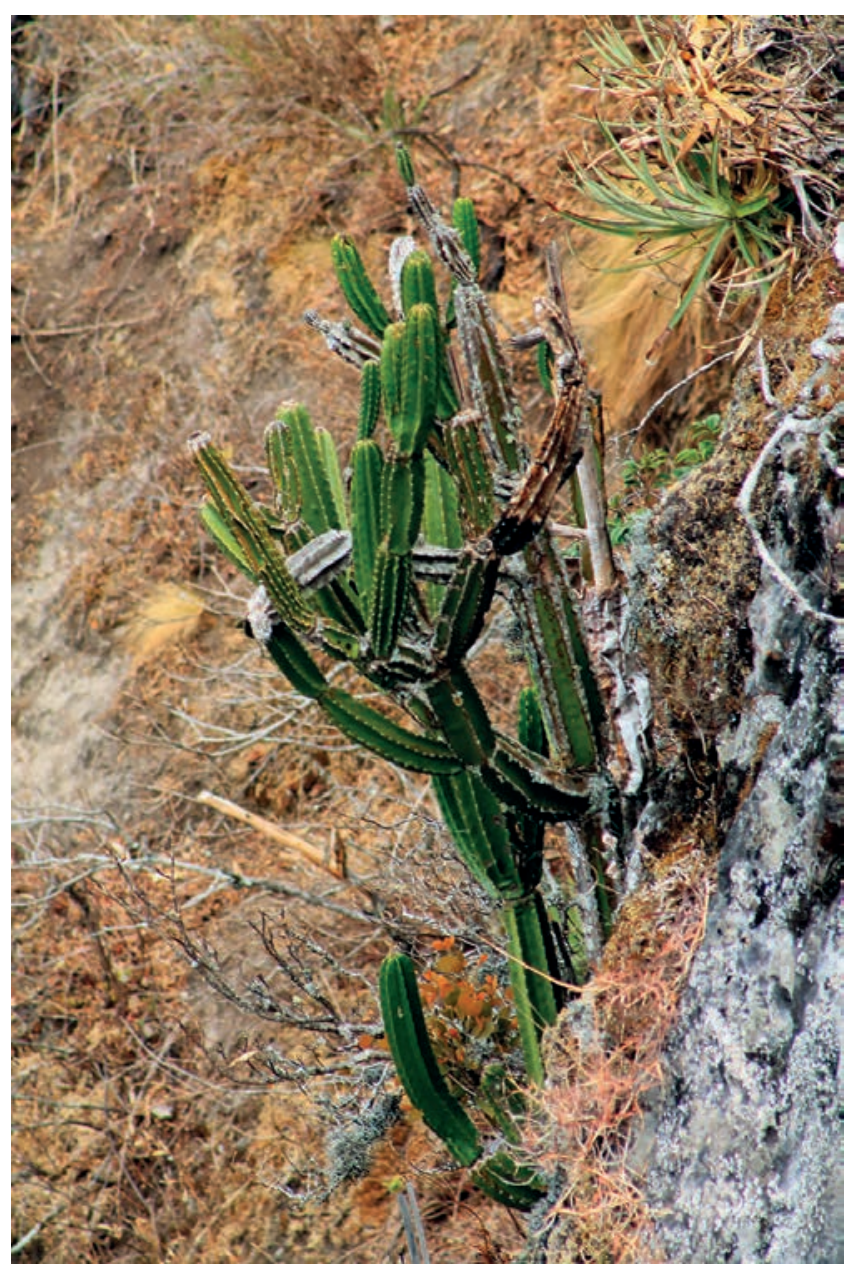

Figura 4. Armatocereus brevispinus / C. Loaiza (C)

pequeńo, de color café - amarillento. Las semillas son numerosas, de forma ovoide - reniforme y de color negro (Madsen 1989, Loaiza 2013).

Estado taxonómico.- Madsen y Aguirre (2004) sinonimizan a esta especie bajo un nombre antiguo, considerándola como $A$. rupicola, en base a ciertas características presentadas en Ritter (1981). El criterio taxonómico utilizado por Madsen y Aguirre (2004) no puede ser considerado como válido por cuanto la descripción de la especie señalada por Ritter no concuerda con las características observadas en las poblaciones presentes en la provincia de Loja (Loaiza 2010, 2013). La especie descrita como A. rupicola y mencionada tanto por Ritter (1981) y Ostolaza (2006) se caracteriza por sus espinas rectas y fuertes, de color negro o gris oscuro; un mayor número de espinas centrales ( 4 - 8), de $2-6 \mathrm{~cm}$ de largo, y también por un mayor número de espinas radiales $(10$ - 12), de $2-6 \mathrm{~mm}$ de largo. La única característica que ambas especies presentan en común es su hábitat, asociado a zonas rocosas y arbustivas. Además, biogeográficamente se ha establecido que $A$. brevispinus se encuentra situado dentro de un trazo generalizado (distrito) denominado como "Trazo interandino del sur" (Loaiza \& Morrone 2011), el cual se ubica entre las provincias de Loja, Azuay y parte de la provincia de Cañar (Ecuador), incluyendo también a ciertos valles secos interandinos del Departamento de Piura (Perú), lo cual deja a $A$. rupicola en una zona bastante alejada a nivel geográfico haciendo que sea improbable una conexión entre ambos taxas.
Se presenta una clave de identificación para algunas especies del género Armatocereus, la cual fue diseñada a partir de individuos adultos, tomando en cuenta sus patrones de coloración, así como las medidas morfométricas de cada especie y otros tipos de estructuras que puedan estar ausentes o ser muy pocos desarrolladas en individuos jóvenes:

\section{Género Armatocereus}

Plantas columnares y ramificadas, divididas en segmentos; brotes, flores y frutos recubiertos por espinas de forma y tamaño variable. Espinas de tipo radial, con $1-4$ espinas centrales y varias espinas radiales según la especie.

1a Espina central reducida (6 - $7 \mathrm{~mm}$ de long.), espinas radiales inconspicuas, 7 a 10 por areola:

A. brevispinus

1b Espinas centrales y radiales conspicuas, tamaño superior a $1 \mathrm{~cm}$ de longitud.

2a Espinas radiales $(9-13)$ por areola, anguladas y aplanadas; flores con los sépalos pubescentes; frutos recubiertos por espinas de color café rojizo:

A. laetus

2b Espinas radiales $(15$ - 25) por areola; tallos maderables, segmentos del tallo de color verde oscuro; flores con los sépalos desnudos, sin pelos (glabros).

3a Brácteas florales de $0.5-1 \mathrm{~mm}$ de long.; areolas florales de $1-2 \mathrm{~mm}$ de ancho, espinas centrales $(1-4)$, de $1-15 \mathrm{~cm}$ de long.; frutos recubiertos por espinas de color amarillento: $A$. cartwrightianus

3b Brácteas florales de $1-5 \mathrm{~mm}$ de long.; areolas florales de $2-5 \mathrm{~mm}$ de ancho; espina central de $1-5 \mathrm{~cm}$ de long.; frutos recubiertos por espinas de color amarillento:

A. godingianus

Distribución y biogeografía.- El modelamiento realizado en Maxent para esta especie tuvo un nivel de aceptación bastante óptimo (Fig. 5). El valor de AUC obtenido fue 0.999 y el grado de predicción al azar de 0.5 , lo cual indica un nivel de confianza bastante aceptable (Fig. 6).

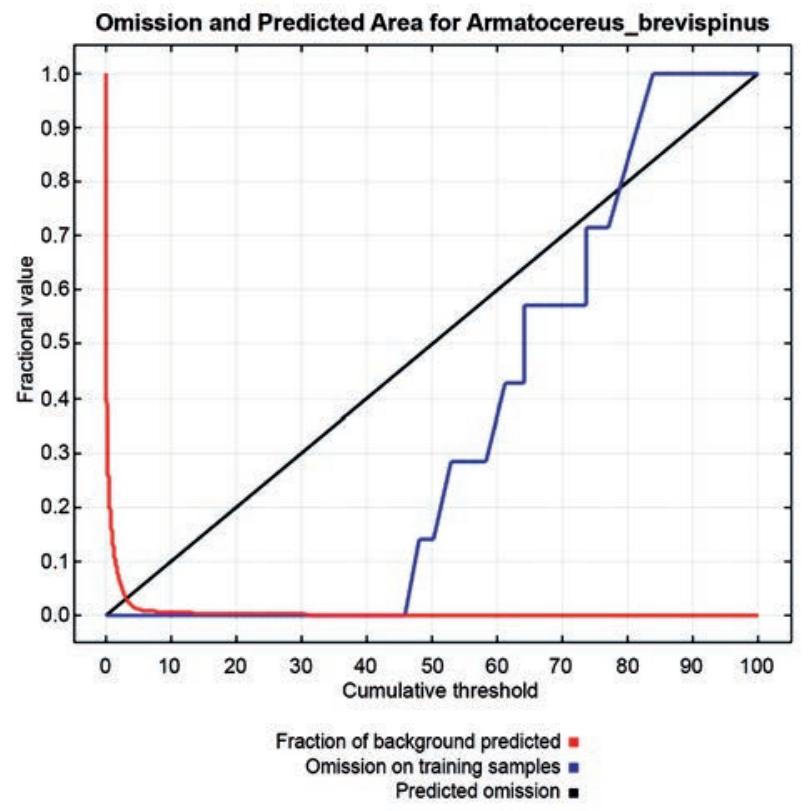

Figura 5: Modelo de curva acumulativa (AUC) para Armatocereus brevispinus 


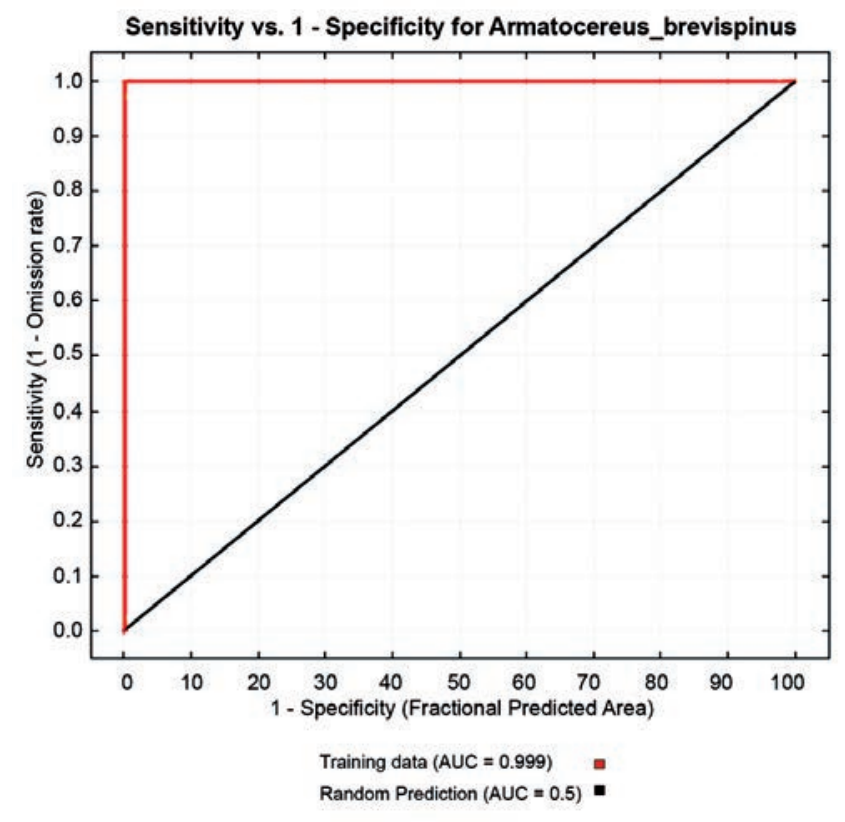

Figura 6. Valor de AUC obtenido para Armatocereus brevispinus.
Tabla 2. Porcentaje de contribución (\% C) y importancia de la permutación $(\mathrm{PI})$ de cada variable para el modelamiento de Armatocereus brevispinus.

\begin{tabular}{lcll}
\hline \multirow{2}{*}{ Variables } & Descripción & \multicolumn{2}{c}{ Modelamiento } \\
& & NC C & PI \\
\hline cld6190_ann & Nubosidad anual & 23.3 & 18.3 \\
dtr6190_ann & Rango de temperatura diurna anual & 47.8 & 72.8 \\
ecoreg & Vegetación potencial (tipo) & 0.6 & 0 \\
frs6190_ann & Frecuencia de heladas anual & 7.3 & 1.4 \\
h_dem & Modelo digital de elevación & 0 & 0 \\
pre6190_ann & Precipitación anual & 0 & 0 \\
pre6190_11 & Precipitación del mes de Enero & 13.6 & 5.7 \\
pre6190_110 & Precipitación del mes de Octubre & 2.4 & 0.2 \\
pre6190_14 & Precipitación del mes de Abril & 0.4 & 0.3 \\
pre6190_17 & Precipitación del mes de Julio & 2.6 & 1.2 \\
tmn6190_ann & Temperatura media anual & 2 & 0 \\
tmp6190_ann & Temperatura mínima anual & 0 & 0 \\
tmx6190_ann & Temperatura máxima anual & 0 & 0 \\
vap6190_ann & Presión de vapor anual & 0 & 0 \\
Total & & 99 & - \\
\hline
\end{tabular}

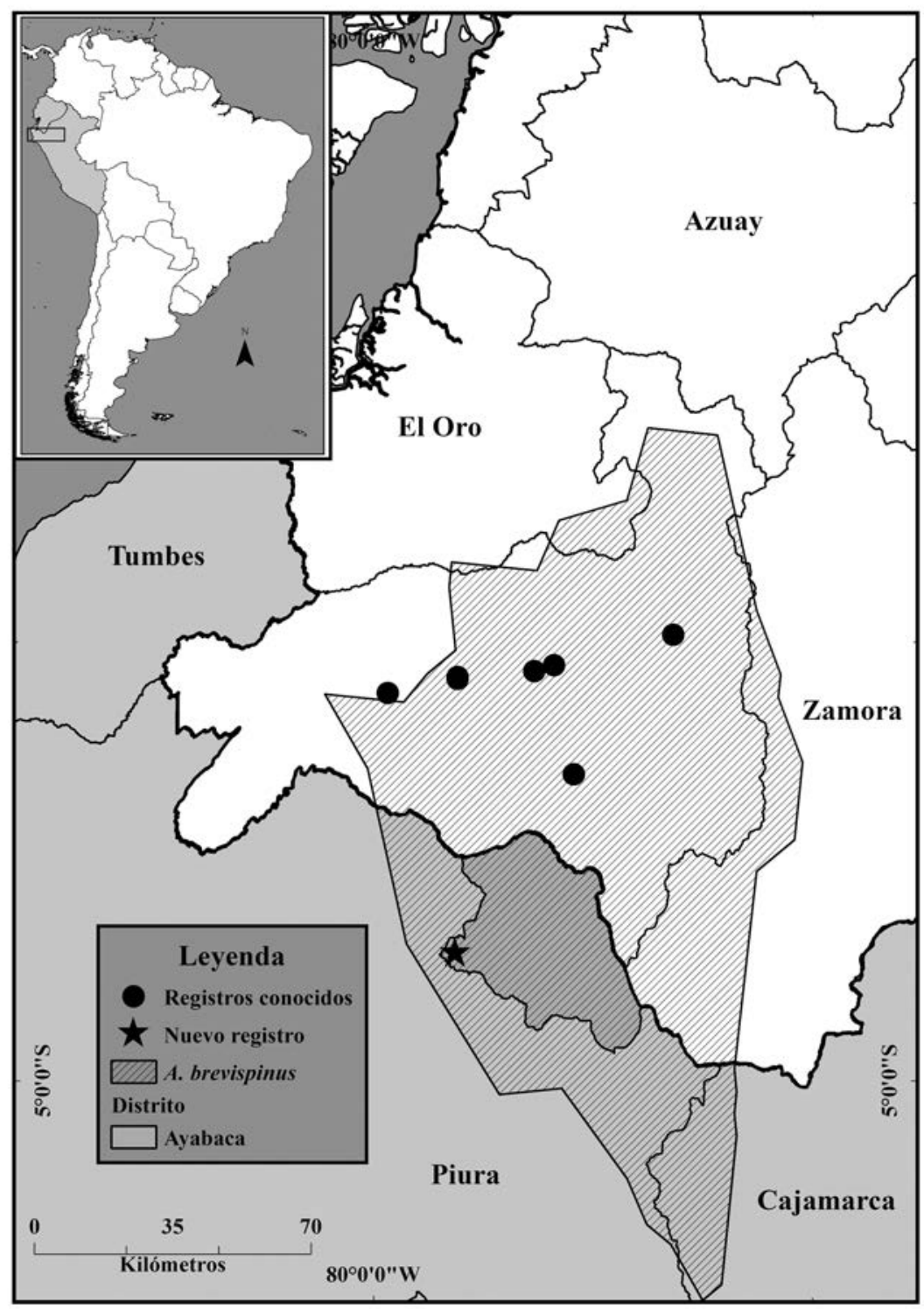

Figura 7. Mapa de distribución de Armatocereus brevispinus. 
De las variables utilizadas, 7 variables con valores superiores al $1 \%$ contribuyeron con el modelamiento realizado, lo cual representa el 99\% de la varianza explicada (Tabla 2). Las variables con mayor influencia para la distribución de $A$. brevispinus fueron: dtr6190_ann (47.8\%), cld6190_ann (23.3\%) y pre6190_11 (13.6\%).

El nuevo registro señalado en el presente trabajo permite confirmar la hipótesis que se tenía sobre la probable presencia de esta especie en el norte de Perú (Loaiza 2013). En base a ello, esta especie pasaría a integrar el grupo de especies endémicas de la región tumbesina. Su distribución actual por lo tanto comprende la región sur del Ecuador y norte del Perú (Fig. 7).

El tipo de formaciones vegetales en las cuales se distribuye esta especie corresponden a Bosque deciduo piemontano y Matorral seco montano (Valencia et al. 1999). Su rango altitudinal se sitúa entre los 800 - $2000 \mathrm{~m}$ de altitud (Herbarios: HUTPL, LOJA, USM, QCA y QCNE). Esta especie se encuentra situada dentro del trazo generalizado (distrito) denominado como "Trazo interandino del sur" (Loaiza y Morrone 2011), el cual se ubica entre las provincias de Loja, Azuay y parte de la provincia del Cañar (Ecuador), incluyendo también algunos valles secos interandinos y varias zonas de matorral seco espinoso en el Departamento de Piura (Perú). Su área de vida corresponde principalmente a valles secos, vertientes rocosas y zonas de matorral seco (Loaiza 2013, Madsen 2002). Esta especie suele formar asociaciones con otras especies de cactus como Opuntia pestifer y O. quitensis (Loaiza 2013). Se encuentra muy poco representada en las colecciones de herbarios tanto de Ecuador como de Perú, razón por la cual ha resultado un poco difícil el poder determinar su correcta distribución, así como también evaluar su estado de conservación. Colecciones adicionales de esta especie, provenientes de las mismas localidades en Ecuador, se encuentran depositadas también en los herbarios AAU, MO y ZSS.

\section{Discusión}

La biogeografía y distribución de cactáceas en los bosques secos del sur de Ecuador y norte de Perú ha sido un tema muy poco estudiado debido a la falta de especialistas en el área y también porque la mayoría de botánicos responsables de las colecciones realizadas a mediados de la década de los 70 y 80 , solo se limitaban a depositar sus colecciones en los herbarios, dejando la responsabilidad de los análisis biogeográficos a otros especialistas, la mayoría de ellos extranjeros, los cuales en la mayoría de los casos poco o nada sabían sobre las condiciones geográficas y formaciones vegetales existentes en este tipo de ecosistemas, por lo cual sus análisis distaban mucho de la realidad.

Actualmente, varios investigadores han dedicado sus esfuerzos al estudio y análisis biogeográfico de la flora de los bosques secos de esta región (Morrone 2001, Sarmiento 1975, Weigend 2002, 2004). Un estudio reciente realizado por Loaiza y Morrone (2011) propone un sistema de clasificación que delimita tres regiones geográficas (distritos) que permiten establecer o delimitar un área florística exclusiva dentro de cada distrito.

El distrito interandino del sur probablemente tendría una distribución prolongada hacia ciertas zonas del norte del Perú, siendo interrumpido por la presencia de barreras geográficas que habrían limitado la libre distribución de las especies, promoviendo en algunos casos la formación de subespecies dentro de un proceso vicariante (Loaiza \& Morrone 2011).
Estas mismas conclusiones y resultados, aunque con otro grupo de plantas también han sido analizadas y discutidas ampliamente por Weigend (2002, 2004). La depresión de Huancabamba sería por lo tanto la barrera geográfica que limitaría la libre distribución de especies hacia el centro y sur del Perú, señalándose como su límite norte, la cuenca del río Jubones en el Ecuador. El caso de Armatocereus brevispinus, sería una de las tantas especies presentes en este distrito o zona biogeográfica, cuya área de distribución específica estaría delimitada en el mapa señalado (Fig. 7), lo cual permite afirmar definitivamente la validez taxonómica de esta especie.

Estado de conservación.- Esta especie estaba considerada dentro de la categoría de especies en peligro crítico $(\mathrm{Cr})$ según el Libro rojo de plantas endémicas del Ecuador (Valencia et al. 2000). La segunda edición del libro rojo de plantas endémicas del Ecuador no incluye esta especie (Madsen \& Montúfar 2011), dadas las consideraciones taxonómicas señaladas por Madsen y Aguirre (2004). El área en la cual fue registrado el holotipo de esta especie por Madsen (1989) actualmente se encuentra bastante alterada y con graves perturbaciones constantes de hábitat. Su restringida distribución en Ecuador y Perú, y su baja densidad poblacional, ponen en grave riesgo la supervivencia de esta especie. Es probable que existan otras poblaciones no registradas en hábitats similares dentro de la provincia de Loja, como por ejemplo en los alrededores del cerro Shiriculapo (Paltas), y en otros cantones como Sozoranga y Gonzanamá. Como medidas de conservación se recomienda realizar monitoreos constantes a las poblaciones existentes y realizar estudios que permitan delimitar su área de distribución en el norte de Perú y establecer el estado actual de su población, así como también realizar estudios enfocados a conocer la biología reproductiva de esta especie, información que permitirá establecer su estado de conservación; además, se debe analizar la posibilidad de la creación de algún tipo de programa de conservación binacional en base a la información obtenida.

\section{Agradecimientos}

Los autores agradecen a la Dra. Haydeé Montoya, jefa del Herbario San Marcos, por su autorización y las facilidades prestadas para la revisión y registro fotográfico del ejemplar USM 28347.

\section{Literatura citada}

Arakaki M. 2011. Contemporaneus and recent radiations of the world's major succulent plant lineages. Proceedings of the National Academy of Sciences 108 (20): 8379-8384. doi: 10.1073/ pnas. 1100628108

Backeberg C. 1938. Blatter fur kakteenforschung 6: 21.

Brack - Egg E. 1986. Las ecorregiones del Perú. Boletín de Lima 44: $57-70$

Hunt D. 2002. Notulae Systematicae Lexicon Cactacearum Spectantes II. Cactaceae Systematics Initiatives 14: 7-11.

Loaiza C.R. 2010. Consideraciones taxonómicas sobre algunas cactáceas nativas de la región sur del Ecuador. Boletín de la Sociedad Latinoamericana y del Caribe de Cactáceas y otras Suculentas 7 (3): 1-8.

Loaiza C.R. 2013. Revisión del género Armatocereus (Cactaceae) en el Ecuador: Biogeografía, distribución y conservación en el Ecuador. Titulación de Biología. Universidad Técnica Particular de Loja / Escuela de Ciencias Biológicas y Ambientales. Loja - Ecuador.

Loaiza C.R. \& J.J. Morrone. 2011. Análisis panbiogeográfico de algunas Cactaceae del Ecuador. Gayana Botánica 68 (1): 1-10. doi: http://dx.doi.org/10.4067/S0717-66432011000200013 
Madsen J.E. 1989. Cactaceae. En G. Harling y L. Anderson (eds.), Flora of Ecuador 35: 1-79.

Madsen J.E. 2002. Cactus en el sur del Ecuador. pp. 289-303 en: Aguirre, Z. et al. Botánica Austroecuatoriana: Estudios sobre los Recursos Vegetales en las Provincias de El Oro, Loja y Zamora Chinchipe. Primera Edición. Ediciones Abya - Yala. Quito, Ecuador.

Madsen J.E. \& Z. Aguirre. 2004. Cactus novelties from southern Ecuador. Nordic Journal of Botany 23 (1): 21-29. doi: 10.1111/j.1756-1051.2003.tb00365.x

Madsen J.E. \& R. Montúfar. 2011. Cactaceae. Págs. 249-252, en: León - Yánez, S., R. Valencia, N. Pitman, L. Endara, C. Ulloa y H. Navarrete (Eds.). 2011. Libro Rojo de las Plantas Endémicas del Ecuador. Segunda Edición. Publicaciones del Herbario QCA, Pontificia Universidad Católica del Ecuador, Quito.

Merow C., M.J. Smith \& J.A. Silander. 2013. A practical guide to Maxent for modeling species' distributions: what it does, and why inputs and settings matter. Ecography 36:1058-1069. doi: $10.1111 /$ j.1600-0587.2013.07872.x

Morales N. 2012. Modelos de distribución de especies: Software Maxent y sus aplicaciones en conservación. Revista Conservación Ambiental 2(1): 1-3.

Morrone J. J. 2001. Biogeografía de América Latina y el Caribe. Manuales y Tesis. Zaragoza. Sociedad Entomológica Aragonesa 3, 148 pp.

Nyffeler R. 2002. Phylogenetic relationships in the cactus family (Cactaceae) based on evidence from TRNK/MATK and TRNL/TRNF sequences. American Journal of Botany 89 (2): 312-326. doi: 10.3732/ajb.89.2.312

Ostolaza C. 2006. El género Armatocereus Backeberg. Zonas Áridas 10: $144-154$.

Ostolaza C. 2014. Todos los cactus del Perú. Lima, Perú. 538 Pp.

Phillips S.J., R.P. Anderson \& R.E. Schapire. 2006. Maximum entropy modeling of species geographic distributions. Ecological Modeling 190: 231-259. doi: 10.1016/j.ecolmodel.2005.03.026
Phillips S.J. \& M. Dudík. 2008. Modeling of species distributions with Maxent: new extensions and a comprehensive evaluation. Ecography 31: 161-175. doi: 10.1111/j.09067590.2008.5203.x

Riquelme J.D. 2013. Técnicas para el análisis de patrones de uso del espacio en felinos americanos (Mammalia: Carnivora). Gestión Ambiental 25: 53-84. http://ceachile.cl/revista/ ARCHIVOS\%20PDF/6_GA_25_2013_Riquelme.pdf

Ritter F. 1981. Kakteen in Sudamerika 4: 1270-1278.

Sarmiento G. 1975. The dry plant formations of South America and their floristic connections. Journal of Biogeography 2 (4): 233-251. doi: 10.2307/3037998

Simmons N.B. \& A.L. Wetterer. 2002. Phylogeny and convergence in cactophilic bats. in: Fleming, T.H. \& A. Valiente - Banuet, eds. Columnar cacti and their mutualists: Evolution, ecology, and conservation. Chapter 5. The University of Arizona Press, Tucson. Pp. 87-93.

Valencia R.N., C. Cerón, W. Palacios \& R. Sierra. 1999. Las formaciones naturales de la Sierra del Ecuador, en: R. Sierra (ed.), Propuesta preliminar de un sistema de clasificación vegetal para el Ecuador Continental. Pp. 79-108. Proyecto INEFAN / GEF - BIRF y EcoCiencia, Quito. 193 pp.

Valencia R.N., N.S. Pitman, S. León-Yánez \& P.M. Jorgensen (Eds.). 2000. Libro rojo de las plantas endémicas del Ecuador. Herbario QCA / Pontificia Universidad Católica del Ecuador. Quito. 489 pp.

Weigend M. 2002. Observations on the biogeography of the Amotape Huancabamba zone in northern Peru. The Botanical Review 68 (1): 38-54. doi: 10.1663/0006-8101(2002)068[0038:OO TBOT]2.0.CO;2

Weigend M. 2004. Observaciones adicionales sobre la biogeografía de la zona de Amotape - Huancabamba en el norte del Perú: definiendo el límite suroriental. Revista peruana de biología 11 (2): 127-134. http://dx.doi.org/10.15381/rpb.v11i2.2447 
